PROCEEDINGS OF THE

AMERICAN MATHEMATICAL SOCIETY

Volume 137, Number 10, October 2009, Pages 3487-3496

S 0002-9939(09)09930-4

Article electronically published on May 20, 2009

\title{
FROM RANDOM WALKS TO ROUGH PATHS
}

\author{
EMMANUEL BREUILLARD, PETER FRIZ, AND MARTIN HUESMANN
}

(Communicated by Peter A. Clarkson)

\begin{abstract}
Donsker's invariance principle is shown to hold for random walks in rough path topology. As an application, we obtain Donsker-type weak limit theorems for stochastic integrals and differential equations.
\end{abstract}

\section{INTRODUCTION}

Consider a random walk in $\mathbb{R}^{d}$, given by the partial sums of a sequence of independent random variables $\left(\xi_{i}: i=1,2,3, \ldots\right)$, identically distributed, $\xi_{i} \stackrel{\mathcal{D}}{=} \xi$ with zero-mean and unit variance. Donsker's theorem (e.g. 11]) states that the rescaled and piecewise linearly connected random walk

$$
W_{t}^{(n)}=\frac{1}{n^{1 / 2}}\left(\xi_{1}+\cdots+\xi_{[t n]}+(n t-[n t]) \xi_{[n t]+1}\right)
$$

converges weakly to $d$-dimensional Brownian motion $B$ in the sense that

$$
\mathbb{E}\left(f\left(W^{(n)}\right)\right) \rightarrow \mathbb{E}(f(B)) \text { as } n \rightarrow \infty,
$$

for all continuous, bounded functionals $f$ on $C\left([0,1], \mathbb{R}^{d}\right)$ with uniform topology. We shall use the shorter notation

$$
W^{(n)} \Longrightarrow B \text { on } C\left([0,1], \mathbb{R}^{d}\right)
$$

to describe this type of convergence. It was observed by Lamperti in [6] that this convergence takes place in $\alpha$-Hölder topology; i.e.

$$
W^{(n)} \Longrightarrow B \text { on } C^{\alpha-\mathrm{Höl}}\left([0,1], \mathbb{R}^{d}\right)
$$

for $\alpha<(p-1) / 2 p$ provided $\mathbb{E}\left(|\xi|^{2 p}\right)<\infty, p>1$ and the condition relating $\alpha$ and $p$ is sharp. In particular, for convergence in $\alpha$-Hölder topology for any $\alpha<1 / 2$ one needs finite moments of any order.

Since $t \mapsto W_{t}^{(n)}$ is a (random) Lipschitz path, it can be canonically lifted by computing iterated integrals up to any given order, say $N$. The resulting lifted path is denoted by

$$
t \mapsto S_{N}\left(W_{t}^{(n)}\right) \equiv\left(1, W_{t}^{(n)}, \int_{0}^{t} W_{s}^{(n)} \otimes W_{s}^{(n)}, \ldots\right)
$$

Received by the editors October 16, 2008.

2000 Mathematics Subject Classification. Primary 60B15.

Key words and phrases. Donskers's theorem, rough paths. 
and can be viewed as a (random) path with values in the step- $N$ free nilpotent group, realized as the subset $G^{N}\left(\mathbb{R}^{d}\right)$ of the tensor algebra $\mathbb{R} \oplus \mathbb{R}^{d} \oplus \cdots \oplus\left(\mathbb{R}^{d}\right)^{\otimes N}$. See [2] and 17, 9, 10] for background on iterated integrals and rough paths. One can ask if $S_{N}\left(W^{(n)}\right)$ converges weakly to a limit. The obvious candidate is Brownian motion $\mathbf{B}$ on the step- $N$ free nilpotent group, i.e. the symmetric diffusion with the generator given by the sub-Laplacian on the stratified Lie group $G^{N}\left(\mathbb{R}^{d}\right)$ associated to the covariance matrix of $\boldsymbol{\xi}$. In uniform topology, the answer is affirmative and follows from work of Stroock-Varadhan [12]; see also [13].

There is motivation from rough path theory to work in stronger topologies than the uniform one. Indeed, various operations of SDE theory and stochastic integration theory are continuous functions of enhanced Brownian motion (i.e. Brownian motion plus Lévy's area or, equivalently, Brownian motion on the step-2 free nilpotent group) in the rough path sense. More specifically, any $G^{2}\left(\mathbb{R}^{d}\right)$-valued path $\mathbf{x}(\cdot)$ has canonically defined path increments, denoted by $\mathbf{x}_{s, t}:=\mathbf{x}_{s}^{-1} \mathbf{x}_{t}$, so that $\left\|\mathbf{x}_{s, t}\right\|=d\left(\mathbf{x}_{s}, \mathbf{x}_{t}\right)$, where $\|\cdot\|$ and $d$ are the (Euclidean) Carnot-Caratheodory norm and metric on $G^{2}\left(\mathbb{R}^{d}\right)$. In its simplest (non-trivial) setting, and under mild regularity conditions on the vector fields $V_{1}, \ldots, V_{d}$, rough path theory asserts that the ODE solution to

$$
d y=\sum_{i=1}^{d} V_{i}(y) d x^{i}, y(0)=y_{0} \in \mathbb{R}^{e},
$$

depends continuously (uniformly on bounded sets) on the driving signal $x(\cdot) \in$ $C^{\infty}\left([0,1], \mathbb{R}^{d}\right)$ with respect to 1$]$

$$
\tilde{d}_{\alpha \text {-Höl }}\left(x, x^{\prime}\right):=d_{\alpha \text {-Höl }}\left(S_{2}(x), S_{2}\left(x^{\prime}\right)\right), \alpha \in(1 / 3,1 / 2),
$$

where $\mathbf{x}:=S_{2}(x)$ and

$$
d_{\alpha-\text { Нӧl }}\left(\mathbf{x}, \mathbf{x}^{\prime}\right)=\sup _{s, t \in[0,1]} \frac{d\left(\mathbf{x}_{s, t}, \mathbf{x}_{s, t}^{\prime}\right)}{|t-s|^{\alpha}} .
$$

It is easy to see that 2

$$
C^{\alpha-\mathrm{Höl}}\left([0,1], G^{2}\left(\mathbb{R}^{d}\right)\right):=\left\{\mathbf{x}: d_{\alpha-\mathrm{Höl}}(\mathbf{x}, \mathbf{1})<\infty\right\}
$$

is a complete metric space for $d_{\alpha \text {-Höl }}$ (up to constants : $d_{\alpha \text {-Höl }}\left(\mathbf{x}, \mathbf{x}^{\prime}\right)=0$ iff $\mathbf{x}_{t}^{-1} \mathbf{x}_{t}^{\prime} \equiv$ constant). It follows that the very meaning of the ODE (1.1) can be extended, in

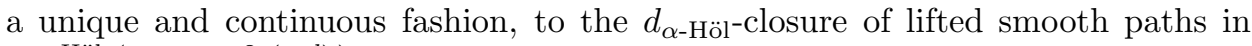
$C^{\alpha \text {-Höl }}\left([0,1], G^{2}\left(\mathbb{R}^{d}\right)\right)$. This closure is a Polish space for the metric $d_{\alpha \text {-Höl }}$ and is denoted by

$$
C^{0, \alpha-\mathrm{Höl}}\left([0,1], G^{2}\left(\mathbb{R}^{d}\right)\right) .
$$

If $\alpha \in(1 / 3,1 / 2)$ it is known as the space of geometric $\alpha$-Hölder rough paths 3 It includes almost every realization of enhanced Brownian motion $\mathbf{B}$,

$$
\mathbf{B} \equiv\left(1, B, \int B \otimes \circ d B\right)
$$

\footnotetext{
${ }^{1}$ It is crucial to have $\alpha>1 / 3$. The solution map $x \mapsto y$ would not be continuous in $d_{\alpha \text {-Höl for }}$ $\alpha \leq 1 / 3$.

${ }^{2} \mathbf{1}$ denotes the trivial path identically equal to the unit element $\mathbf{1} \in G^{2}\left(\mathbb{R}^{d}\right)$.

3 The same construction applies when $G^{2}\left(\mathbb{R}^{d}\right)$ is replaced by $G^{N}\left(\mathbb{R}^{d}\right)$, in which case one requires $\alpha \in(1 /(N+1), 1 / N)$ in order to speak of geometric $\alpha$-Hölder rough paths.
} 
where $\circ d B$ denotes the Stratonovich differential of $B$. The resulting "generalized" ODE solution driven by $\mathbf{B}$ can then be identified as the classical Stratonovich SDE solution [7, 9, 10]. This provides an essentially deterministic approach to SDE theory with numerous benefits when it comes to regularity questions of the Itô map, construction of stochastic flows, etc. Another property of such rough paths is that there is a unique (again: modulo constants) lift of $\mathbf{x} \in C^{0, \alpha-\text { Höl }}\left([0,1], G^{2}\left(\mathbb{R}^{d}\right)\right)$ to a path of similar regularity in the step- $N$ group for all $N \geq 2$,

$$
S_{N}(\mathbf{x}) \in C^{0, \alpha-\text { Höl }}\left([0,1], G^{N}\left(\mathbb{R}^{d}\right)\right) .
$$

(See [10, Thm. 3.7] for instance.) In the case of the enhanced Brownian motion $\mathbf{x}=\mathbf{B}(\omega)$, the process $S_{N}(\mathbf{B})$ is identified as Brownian motion $B$ plus all iterated Stratonovich integrals up to order $N$. In particular, $S_{N}(\mathbf{B})$ is then a realization of the Brownian motion on the step- $N$ free nilpotent group.

Our main result is

Theorem 1. Assume $\mathbb{E}\left(|\xi|^{2 p}\right)<\infty$ for some real number $p \geq 4$. Then

$$
S_{2}\left(W_{t}^{(n)}\right) \Longrightarrow \mathbf{B} \text { in } C^{0, \alpha-H o ̈ l}\left([0,1], G^{2}\left(\mathbb{R}^{d}\right)\right),
$$

provided

$$
\alpha \in\left(\frac{1}{3}, \frac{p^{*}-1}{2 p^{*}}\right) \text { with } p^{*}=\min ([p], 2[p / 2]) .
$$

In particular, if $\mathbb{E}\left(|\xi|^{2 p}\right)<\infty$ for all $p<\infty$, then the weak convergence holds for any $\alpha<1 / 2$.

Let us point out that Theorem 1 implies, of course, $\alpha$-Hölder convergence for all $\alpha<\frac{p^{*}-1}{2 p^{*}}$, but only for $\alpha>1 / 3$ do we actually get the interesting corollaries regarding convergence in rough path topology. Let us also remark that weak convergence of $S_{N}\left(W_{t}^{(n)}\right)$ for all $N$ is a (deterministic) consequence of Theorem 1; cf. Corollary 3 below.

Although reminiscent of Lamperti's sharp upper bound on the Hölder exponent, $\frac{p-1}{2 p}$, the actual "coarsened" form of our upper bound in (1.2), with $p$ replaced by $p^{*}$, i.e. the largest even integer smaller than or equal to $p$, comes from our argument (which requires us to work with integer powers). To handle the case of "integrability level" $p \in(1,4)$ it is clear that the step-2 setting will not be sufficient. Indeed, from Lamperti's bound, we would have to work at least in the step- $N$ group with $N \sim 2 p /(p-1)$. More precisely, we would need to be able to work in $\alpha$-Hölder topology for $G^{N}\left(\mathbb{R}^{d}\right)$-valued paths with

$$
1 /(N+1)<\alpha<(p-1) / 2 p .
$$

Any result of the form

$$
S_{N}\left(W_{t}^{(n)}\right) \Longrightarrow S_{N}(\mathbf{B}) \text { in } C^{0, \alpha-\mathrm{Höl}}\left([0,1], G^{N}\left(\mathbb{R}^{d}\right)\right)
$$

would then be equally interesting, as it would constitute a "step- $N$ " convergence result in rough path topology with similar corollaries as those described below. Unfortunately, as explained in Section 4 , the "coarsened" Hölder exponents that we obtain in the step- $N$ setting are not larger than $1 /(N+1)$ in general. Although we suspect this to be an artifact of our proof, we currently do not know how to bypass this difficulty in order to handle $p \in(1,4)$. 
Let us now discuss some applications to Theorem 1. The aforementioned continuity results of rough path theory lead immediately to corollaries of the following type (recall that we assume existence of a moment of order $p \geq 4$ for the $\boldsymbol{\xi}_{i}$ 's).

Corollary 1 (Donsker-Wong-Zakai-type convergence). Assume $V=\left(V_{1}, \ldots, V_{d}\right)$ is a collection of $C^{3}$-bounded vector fields on $\mathbb{R}^{e}$ and let $\left(Y^{n}\right)$ denote the family of (random) ODE solutions to

$$
d Y^{n}=V\left(Y^{n}\right) d W^{(n)}, \quad Y_{0}=y_{0} \in \mathbb{R}^{e} .
$$

Then, with $\alpha$ as in (1.2),

$$
Y^{n} \Longrightarrow Y \text { in } C^{\alpha-H \ddot{o l} l}\left([0,1], \mathbb{R}^{e}\right),
$$

where $Y$ is the (up to indistinguishability) unique continuous solution to the Stratonovich SDE

$$
d Y=V(Y) \circ d B, \quad Y_{0}=y_{0} \in \mathbb{R}^{e} .
$$

Remark 1 . The regularity assumptions of the $\left(V_{i}\right)$ can be slightly weakened. One can also add a drift vector field (only assumed $C^{1}$-bounded, say), and in fact the weak convergence can be seen to hold in the sense of flows of $C^{1}$-diffeomorphisms (and then $C^{k}$-flows for $k \in \mathbb{N}$, provided additional smoothness assumptions are made on $V$ ). Indeed, all this follows from the appropriate (deterministic) continuity results of rough path theory (cf. for instance [4) combined with stability of weak convergence under continuous maps.

Corollary 2 (Weak convergence to stochastic integrals). Assume $\varphi=\left(\varphi_{1}, \ldots, \varphi_{d}\right)$ is a collection of $C_{b}^{2}$-bounded functions from $\mathbb{R}^{d}$ to $\mathbb{R}^{e}$. Then

$$
\int_{0}^{\cdot} \varphi\left(W^{(n)}\right) d W^{(n)} \Longrightarrow \int_{0}^{\cdot} \varphi(B) \circ d B \text {. }
$$

Corollary 3 (Convergence to BM on the free step- $N$ nilpotent group). Assume $N \geq 2$. Then

$$
S_{N}\left(W_{t}^{(n)}\right) \Longrightarrow \tilde{\mathbf{B}} \text { in } C^{0, \alpha-H o ̈ l}\left([0,1], G^{N}\left(\mathbb{R}^{d}\right)\right)
$$

with $\alpha$ as in (1.2) and $\tilde{\mathbf{B}}$ a Brownian motion on $G^{N}\left(\mathbb{R}^{d}\right)$.

As a final remark, let us note that some illustrative examples of random walks from a rough path point of view (but no definite theorems) have been given previously in [8]. For instance, the rough path convergence of Brownian motion and its $\varepsilon$-delay (due to B. Hoff; cf. [8]) is easily understood from a random walk point of view.

\section{Donsker's THEOREM For ENHANCED Brownian MOTION AND RANDOM WALKS ON GROUPS}

We first discuss the case of a random walk for finite moments of all orders.

Theorem 2 (Donsker's theorem for enhanced Brownian motion). Assume $\mathbb{E} \xi=0$ and $\mathbb{E}\left(|\xi|^{p}\right)<\infty$ for all $p \in[1, \infty)$ and $\alpha<1 / 2$. Then

$$
S_{2}\left(W^{(n)}\right) \Longrightarrow \mathbf{B} \text { in } C^{0, \alpha-H \ddot{o} l}\left([0,1], G^{2}\left(\mathbb{R}^{d}\right)\right),
$$

where $\mathbf{B}$ is a $\left(G^{2}\left(\mathbb{R}^{d}\right)\right.$-valued $)$ enhanced Brownian motion. 
In fact, we shall prove a more general theorem that deals with random walks on groups. More precisely, by a theorem of Chen [2] we have

$$
S_{2}\left(W^{(n)}\right)_{t}=\delta_{n^{-1 / 2}}\left(e^{\xi_{1}} \otimes \cdots \otimes e^{\xi_{[n t]}} \otimes e^{(n t-[n t]) \xi_{[n t]+1}}\right),
$$

where $\delta$ denotes dilation on $G^{2}\left(\mathbb{R}^{d}\right)$ and $e^{v}=\left(1, v, v^{\otimes 2} / 2\right) \in G^{2}\left(\mathbb{R}^{d}\right), v \in \mathbb{R}^{d}$. Observe that $\left(\boldsymbol{\xi}_{i}\right):=\left(e^{\xi_{i}}\right)$ is a sequence of independent, identically distributed $G^{2}\left(\mathbb{R}^{d}\right)$-valued random variables centered in the sense that

$$
\mathbb{E}\left(\pi_{1}\left(\boldsymbol{\xi}_{i}\right)\right)=\mathbb{E} \xi_{i}=0,
$$

where $\pi_{1}$ is the projection from $G^{2}\left(\mathbb{R}^{d}\right) \rightarrow \mathbb{R}^{d}$. Let us also observe that the shortest path which connects the unit element $1 \in G^{2}\left(\mathbb{R}^{d}\right)$ with $e^{\xi_{i}}$ is precisely $e^{t \xi_{i}}$, so that piecewise linear interpolation on $\mathbb{R}^{d}$ lifts to geodesic interpolation on $G^{2}\left(\mathbb{R}^{d}\right)$. We shall thus focus on the following Donsker-type theorem.

Theorem 3. Let $\left(\boldsymbol{\xi}_{i}\right)$ be a centered IID sequence of $G^{2}\left(\mathbb{R}^{d}\right)$-valued random variables with finite moments of all orders,

$$
\forall q \in[1, \infty): E\left(\left\|\boldsymbol{\xi}_{i}\right\|^{q}\right)<\infty
$$

and consider the rescaled random walk defined by $\mathbf{W}_{0}^{(n)}=1$ and

$$
\mathbf{W}_{t}^{(n)}=\delta_{n^{-1 / 2}}\left(\boldsymbol{\xi}_{1} \otimes \cdots \otimes \boldsymbol{\xi}_{[t n]}\right)
$$

for $t \in\left\{0, \frac{1}{n}, \frac{2}{n}, \ldots\right\}$, piecewise geodesically connected in between (i.e. $\left.\mathbf{W}_{t}^{(n)}\right|_{\left[\frac{i}{n}, \frac{i+1}{n}\right]}$ is a geodesic connecting $\mathbf{W}_{i / n}^{(n)}$ and $\mathbf{W}_{(i+1) / n}^{(n)}$ ). Then, for any $\alpha<1 / 2, \mathbf{W}^{(n)}$ converges weakly to $\mathbf{B}$, in $C^{0, \alpha-H o ̈ l}\left([0,1], G^{2}\left(\mathbb{R}^{d}\right)\right)$.

\section{Proof of Theorem 3}

Following a standard pattern of proof, weak convergence follows from convergence of the finite-dimensional distributions and tightness (here in $\alpha$-Hölder topology).

Step 1 (Convergence of the finite-dimensional distributions). This is an immediate consequence of

Theorem 4 (Central limit theorem for centered i.i.d. variables on a nilpotent Lie group). Let $N$ be a simply connected nilpotent Lie group and let $\boldsymbol{\xi}_{1}, \ldots, \boldsymbol{\xi}_{n}, \ldots$ be i.i.d. random variables with values in $N$ which we assume centered (i.e. their projection $\pi_{1}\left(\boldsymbol{\xi}_{i}\right)$ on the abelianization of $N$ has zero mean) and with a finite second moment, i.e. $E\left(\left\|\boldsymbol{\xi}_{i}\right\|^{2}\right)<\infty$. Then we have the following convergence in law:

$$
\delta_{n^{-1 / 2}}\left(\boldsymbol{\xi}_{1} \otimes \cdots \otimes \boldsymbol{\xi}_{n}\right) \rightarrow \mathbf{B}_{1},
$$

where $\mathbf{B}_{1}$ is the time 1 value of the Brownian motion on $N$ associated to $\boldsymbol{\xi}_{1}$ (i.e. the symmetric diffusion on $N$ with infinitesimal generator the left invariant subLaplacian $\frac{1}{2} \sum a_{i j} X_{i} X_{j}$, where $\left(a_{i j}\right)$ is the covariance matrix of $\left.\pi_{1}\left(\xi_{i}\right)\right)$.

This theorem is a straightforward consequence of the main result of Wehn's (unpublished) 1962 thesis; cf. [5], [1, Thm. 3.11] or [3]. It also follows a fortiori from the much stronger Stroock-Varadhan Donsker-type theorem in connected Lie groups [12]. 
Step 2 (Tightness). We need to find positive constants $a, b, c$ such that for all $u, v \in[0,1]$,

$$
\sup _{n} \mathbb{E}\left[d\left(\mathbf{W}_{v}^{(n)}, \mathbf{W}_{u}^{(n)}\right)^{a}\right] \leq c|v-u|^{1+b} .
$$

Then we can apply Kolmogorov's tightness criterion 4 to obtain tightness in $\gamma$-Hölder topology, for any $\gamma<b / a$. Using basic properties of geodesic interpolation, we see that it is enough to consider $u, v \in\left\{0, \frac{1}{n}, \frac{2}{n}, \ldots\right\}$ and then (by independence of increments and left invariance of $d$ ) there is no loss of generality in taking $[u, v]=$ $[0, k / n]$ for some $k \in\{0, \ldots, n\}$. It follows that what has to be established reads as

$$
\exists a, b, c_{1}: \frac{1}{n^{a / 2}} E\left[\left\|\boldsymbol{\xi}_{1} \otimes \cdots \otimes \boldsymbol{\xi}_{k}\right\|^{a}\right] \leq c_{1}\left|\frac{k}{n}\right|^{1+b},
$$

uniformly over all $n \in \mathbb{N}$ and $0 \leq k \leq n$ and such that $b / a$ can be taken arbitrarily close to $1 / 2$. To this end, it is enough to show that for all $p \in\{1,2, \ldots\}$

$$
(*): \mathbb{E}\left[\left\|\boldsymbol{\xi}_{1} \otimes \cdots \otimes \boldsymbol{\xi}_{k}\right\|^{4 p}\right]=O\left(k^{2 p}\right)
$$

since we can then take $a=4 p, b=2 p-1$ and of course $b / a=(2 p-1) /(4 p) \uparrow 1 / 2$ as $p \uparrow \infty$. Thus, the proof is finished once we show $(*)$, and this is the content of the last step of this proof.

Step 3. Let $P$ be a polynomial function on $G^{2}\left(\mathbb{R}^{d}\right)$, i.e. a polynomial in $a^{1 ; i}, a^{2 ; i j}$ where

$$
a=\left(a^{1 ; i}, a^{2 ; i j} ; 1 \leq i \leq d, 1 \leq i<j \leq d\right) \in \mathfrak{g}^{2}\left(\mathbb{R}^{d}\right)
$$

is the log-chart of $G^{2}\left(\mathbb{R}^{d}\right), g \mapsto a=\log (g)$. We define the degree $d^{\circ} P$ by agreeing that monomials of the form

$$
\left(a^{1 ; i}\right)^{\alpha_{i}}\left(a^{2 ; i j}\right)^{\alpha_{i, j}}
$$

have degree $\sum \alpha_{i}+2 \sum \alpha_{i, j}$. If $\boldsymbol{\xi}$ is a $G^{2}\left(\mathbb{R}^{d}\right)$-valued random variable with moments of all orders, then

$$
T P: g \mapsto \mathbb{E}(P(g \otimes \boldsymbol{\xi}))-P(g)
$$

is well defined and is another polynomial function. Moreover if $\boldsymbol{\xi}$ is centered, an easy application of the Campbell-Baker-Hausdorff formula reveals that $T P$ is of degree $\leq d^{o} P-2$. (For instance, $P(a):=\left(a^{2 ; i j}\right)^{m}$ has degree $2 m$; then $T P$ is seen to contain terms of the form $\left(a^{2 ; i j}\right)^{m-1}$ and $\left(a^{2 ; i j}\right)^{m-2}\left(a^{1 ; k}\right)^{2}$, etc., all of which are indeed of degree $2 m-2$.) Now, for any $p \in\{1,2, \ldots\}$,

$$
\begin{aligned}
\left\|e^{a}\right\|^{4 p} & \sim \sum_{i}\left|a^{1 ; i}\right|^{4 p}+\sum_{i<j}\left|a^{1 ; i j}\right|^{2 p} \\
& =\sum_{i}\left(a^{1 ; i}\right)^{4 p}+\sum_{i<j}\left(a^{1 ; i j}\right)^{2 p}=: P\left(e^{a}\right),
\end{aligned}
$$

\footnotetext{
${ }^{4}$ E.g. [11; the extension from the real-valued processes to $\left(G^{2}\left(\mathbb{R}^{d}\right), d\right)$-valued processes, with $d$ being the Carnot-Carathéodory metric, is trivial.
} 
where $P$ is a polynomial of degree $4 p$. Recalling the definition of the operator $T$ and using independence, we have

$$
\begin{aligned}
\mathbb{E}\left[P\left(\boldsymbol{\xi}_{1} \otimes \cdots \otimes \boldsymbol{\xi}_{k}\right)\right] & =\mathbb{E}\left[\mathbb{E}\left[P\left(\left(\boldsymbol{\xi}_{1} \otimes \cdots \otimes \boldsymbol{\xi}_{k-1}\right) \otimes \boldsymbol{\xi}_{k}\right) \mid \boldsymbol{\xi}_{1}, \ldots, \boldsymbol{\xi}_{k-1}\right]\right] \\
& =\mathbb{E}\left[T P\left(\boldsymbol{\xi}_{1} \otimes \cdots \otimes \boldsymbol{\xi}_{k-1}\right)+P\left(\boldsymbol{\xi}_{1} \otimes \cdots \otimes \boldsymbol{\xi}_{k-1}\right)\right] \\
& =\cdots \\
& =(T+1)^{k} P(1) \\
& =\sum_{l \geq 0}\left(\begin{array}{l}
k \\
l
\end{array}\right) T^{l} P(1) .
\end{aligned}
$$

But the function $T P: g \mapsto \mathbb{E}(P(g \otimes \boldsymbol{\xi}))-P(g)$ is a polynomial function of degree at most $d^{\circ} P-2=4 p-2$. Hence

$$
d^{\circ} T^{l} P \leq d^{\circ} P-2 l=2(2 p-l),
$$

and the above sum contains only a finite number of terms, more precisely

$$
\mathbb{E}\left[P\left(\boldsymbol{\xi}_{1} \otimes \cdots \otimes \boldsymbol{\xi}_{k}\right)\right]=\sum_{l=0}^{2 p}\left(\begin{array}{c}
k \\
l
\end{array}\right) T^{l} P(1)
$$

Since each of these terms is $O\left(k^{2 p}\right)$, as $k \rightarrow \infty$, we are done.

\section{EXtension to Finite MOMEnts}

The question remains as to what happens if we weaken the moment assumption to

$$
\mathbb{E}\left(\left\|\boldsymbol{\xi}_{i}\right\|^{2 p}\right)<\infty \text { for some } p>1,
$$

where, for now, $\boldsymbol{\xi}_{i} \in G^{2}\left(\mathbb{R}^{d}\right)$. If we could prove that

$$
\mathbb{E}\left[\left\|\boldsymbol{\xi}_{1} \otimes \cdots \otimes \boldsymbol{\xi}_{k}\right\|^{2 p}\right]=O\left(k^{p}\right),
$$

then the arguments of the previous section apply line-by-line to obtain tightness (and hence weak convergence) in $C^{0, \gamma \text {-Höl }}\left([0,1], G^{2}\left(\mathbb{R}^{d}\right)\right)$ for any

$$
\gamma<\frac{p-1}{2 p} \text {. }
$$

In the case where $\frac{p-1}{2 p}>1 / 3$ we can and will choose $\gamma \in(1 / 3,1 / 2)$, since then $C^{0, \gamma \text {-Höl }}\left([0,1], G^{2}\left(\mathbb{R}^{d}\right)\right)$ is a genuine rough path space 5 Otherwise, i.e. if $\gamma<$ $\frac{p-1}{2 p} \leq 1 / 3$, tightness in $C^{0, \gamma \text {-Höl }}\left([0,1], G^{2}\left(\mathbb{R}^{d}\right)\right)$ is worthless (from the point of view of rough path applications). However, we can still ask for the smallest integer $N$ such that

$$
\frac{p-1}{2 p}>\frac{1}{N+1}
$$

and consider $G^{N}\left(\mathbb{R}^{d}\right)$-valued random variables $\boldsymbol{\xi}_{i}$ with finite $(2 p)$-moments. Again, if we could show that (4.1) holds, all arguments would extend and we would obtain tightness in $C^{0, \gamma \text {-Höl }}\left([0,1], G^{N}\left(\mathbb{R}^{d}\right)\right)$, where $\gamma$ can be chosen to be in $\left(\frac{1}{N+1}, \frac{1}{N}\right)$ so that we have tightness in a "step- $N$ rough path topology". Unfortunately, the "polynomial" proof of the previous section does not allow us to obtain (4.1), but only the following slightly weaker result.

\footnotetext{
${ }^{5}$ The integer part of $1 / \gamma$ must match the level of nilpotency: $[1 / \gamma]=2$.
} 
Proposition 1. Let $\left(\boldsymbol{\xi}_{i}\right)$ be a centered IID sequence of $G^{N}\left(\mathbb{R}^{d}\right)$-valued random variables with finite $(2 p)$-moments, $p$ real $p>1$ :

$$
E\left(\left\|\boldsymbol{\xi}_{i}\right\|^{2 p}\right)<\infty
$$

Then

$$
\mathbb{E}\left[\left\|\boldsymbol{\xi}_{1} \otimes \cdots \otimes \boldsymbol{\xi}_{k}\right\|^{2 q}\right]=O\left(k^{q}\right)
$$

for all $q \leq q_{0}(p, N) \leq p$, where $q_{0}(p, N)$ may be taken to be

$$
q_{0}(p, N)=\min _{m=1, \ldots, N} m\left[\frac{p}{m}\right] .
$$

Proof. Set $q_{m}=m[p / m]$. The conclusion is equivalent to

$$
\left|\left\|\boldsymbol{\xi}_{1} \otimes \cdots \otimes \boldsymbol{\xi}_{k}\right\|\right|_{L^{2 q}}=O\left(k^{1 / 2}\right)
$$

and will follow, with $q=\min \left\{q_{m}: m=1, \ldots, N\right\}$, from

$$
\begin{aligned}
& \left.\left.|| \pi_{m}\left(\log \left(\boldsymbol{\xi}_{1} \otimes \cdots \otimes \boldsymbol{\xi}_{k}\right)\right)\right|^{1 / m}\right|_{L^{2 q}} \\
\leq & \left.\left.|| \pi_{m}\left(\log \left(\boldsymbol{\xi}_{1} \otimes \cdots \otimes \boldsymbol{\xi}_{k}\right)\right)\right|^{1 / m}\right|_{L^{2 q_{m}}}=O\left(k^{1 / 2}\right),
\end{aligned}
$$

provided we can show the $O\left(k^{1 / 2}\right)$-estimate of the last line for all $m \in\{1, \ldots, N\}$. To this end, consider $P_{m}\left(e^{a}\right)$ given by

$$
\sum_{i_{1} \ldots i_{m} \in\{1, \ldots, d\}}\left(a^{m ; i_{1} \ldots i_{m}}\right)^{2[p / m]}
$$

and observe that it has homogenous degree $2 m[p / m] \leq 2 p$. Now observe that the condition of existence of moments of order $2 p$ for $\boldsymbol{\xi}$, i.e. (4.2), implies (using Hölder's inequality) that the expectation of any polynomial in $\boldsymbol{\xi}$ of homogeneous degree at most $2 p$ is finite. Hence the operator $T$ defined in Step 3 of the proof of Theorem 3 applies to $P_{m}$, and the argument there (see also [3, Lemma 2.4]) shows that it will reduce its degree by two. The same arguments as earlier will then give us the $O\left(k^{1 / 2}\right)$-estimate with $q_{m}$ determined by

$$
2 q_{m} / m=2[p / m] \text { or } q_{m}=m[p / m] .
$$

As in the previous section, (4.3) with $q=q_{0}$ implies $\alpha$-Hölder tightness/convergence for any $\alpha<\left(q_{0}-1\right) / 2 q_{0}$. In particular, when

$$
\frac{q_{0}-1}{2 q_{0}}>\frac{1}{N+1} \text { or } q_{0}=\min _{m=1, \ldots, N} m\left[\frac{p}{m}\right]>\frac{N+1}{N-1}=1+\frac{2}{N-1}
$$

we can pick $\alpha>\frac{1}{N+1}$ and thus have "rough path convergence".

Case $N=2$. In this case, the above condition reduces to $\min ([p], 2[p / 2])>3$, which requires $p \geq 4$. Indeed, for $p=4$ we have $q_{0}(4, N)=4$, which implies $\alpha$-Hölder tightness/convergence for any

$$
\alpha<\frac{q_{0}-1}{2 q_{0}}=\frac{3}{8}
$$


and since $3 / 8 \in(1 / 3,1 / 2)$, we can indeed pick $\alpha \in(1 / 3,1 / 2)$ such as to have tightness/convergence in rough path topology. Remark that any $p<4$ implies $q_{0}(p, N) \leq 3$ so that we necessarily have to pick

$$
\alpha<\frac{3-1}{6}=\frac{1}{3}
$$

in which case $\alpha$-Hölder topology is not a rough path topology on the space of $G^{N}\left(\mathbb{R}^{d}\right)=G^{2}\left(\mathbb{R}^{d}\right)$-valued paths.

Case $N=3$. In this case, the above condition reduces to $\min ([p], 2[p / 2], 3[p / 3])>2$ which also requires $p \geq 4$. But by assuming $p \geq 4$ we can safely work in the step- 2 group.

Case $N \geq 4$. In this case, $\min ([p], 2[p / 2], 3[p / 3], 4[p / 4], \ldots)>\left[1+\frac{2}{N-1}\right]=1$ so that

$$
m[p / m]>1 \text { and hence } \geq 2
$$

for all $m \in\{1, \ldots, N\}$. In particular we can take $m=4$ and see $4[p / 4] \geq 2 \Longrightarrow$ $[p / 4] \geq 1 \Longrightarrow p \geq 4$. Then again, we can make the remark that under the assumption $p \geq 4$ we can safely work in the step-2 group.

Corollary 4. Assume $\mathbb{E}\left(|\xi|^{2 p}\right)<\infty$ for some real $p \geq 4$. Then the rescaled (step 2) lift of the (rescaled, piecewise linearly connected) random walk $W_{t}^{(n)}$ converges in $\alpha$-Hölder for any

$$
\frac{1}{3}<\alpha<\frac{p^{*}-1}{2 p^{*}} \equiv \alpha^{*}(p),
$$

where $p^{*}=\min ([p], 2[p / 2])$.

Remark 2. For $p \in\{4,6,8, \ldots\}, \alpha^{*}(p)=\frac{p-1}{2 p}$. In particular, $\alpha^{*}(4)=3 / 8$ and

$$
\alpha^{*}(p) \sim \frac{p-1}{2 p} \rightarrow 1 / 2 \text { as } p \rightarrow \infty
$$

in agreement with Theorem 3 .

\section{ACKNOWLEDGMENTS}

The second author was partially supported by a Leverhulme Research Fellowship and EPSRC grant EP/E048609/1. The authors would like to thank an anonymous referee for pointing them to [8].

\section{REFERENCES}

[1] E. Breuillard. Random walks on lie groups. Preprint, 2007.

[2] Kuo-Tsai Chen. Integration of paths, geometric invariants and a generalized Baker-Hausdorff formula. Ann. of Math. (2), 65:163-178, 1957. MR0085251 (19:12a)

[3] Pierre Crépel and Albert Raugi. Théorème central limite sur les groupes nilpotents. Ann. Inst. H. Poincaré Sect. B (N.S.), 14(2):145-164, 1978. MR.507730 (82a:60016)

[4] Peter K. Friz and Nicolas B. Victoir. Multidimensional stochastic processes as rough paths: theory and applications. Cambridge Studies in Advanced Mathematics. Cambridge University Press, Cambridge, 2009, to appear. Draft available at http://www.statslab.cam.ac.uk/ peter/RoughPathsBook/.

[5] Ulf Grenander. Probabilities on algebraic structures. John Wiley \& Sons Inc., New York, 1963. MR0259969 (41:4598) 
[6] John Lamperti. On convergence of stochastic processes. Trans. Amer. Math. Soc., 104:430435, 1962. MR0143245 (26:804)

[7] Terry Lyons. Differential equations driven by rough signals. Rev. Mat. Iberoamericana, 14(2):215-310, 1998. MR1654527(2000c:60089)

[8] Terry Lyons. Systems controlled by rough paths. In European Congress of Mathematics, pages 269-281. Eur. Math. Soc., Zürich, 2005. MR2185750 (2006g:34008)

[9] Terry Lyons and Zhongmin Qian. System Control and Rough Paths. Oxford Mathematical Monographs, Oxford University Press, 2002. MR 2036784 (2005f:93001)

[10] Terry J. Lyons, Michael Caruana, and Thierry Lévy. Differential equations driven by rough paths, volume 1908 of Lecture Notes in Mathematics. Springer, Berlin, 2007. Lectures from the 34th Summer School on Probability Theory held in Saint-Flour, July 6-24, 2004, with an introduction by Jean Picard. MR.2314753 (2009c:60156)

[11] Daniel Revuz and Marc Yor. Continuous martingales and Brownian motion, volume 293 of Grundlehren der Mathematischen Wissenschaften [Fundamental Principles of Mathematical Sciences]. Springer-Verlag, Berlin, third edition, 1999. MR:1725357 (2000h:60050)

[12] Daniel W. Stroock and S. R. S. Varadhan. Limit theorems for random walks on Lie groups. Sankhya Ser. A, 35(3):277-294, 1973. MR0517406 (58:24457)

[13] Joseph C. Watkins. Donsker's invariance principle for Lie groups. Ann. Probab., 17(3):12201242, 1989. MR 1009453 (90m:60013)

[14] Donald Wehn. Probabilities on Lie groups. Proc. Nat. Acad. Sci. U.S.A., 48:791-795, 1962. MR0153042 (27:3011)

Laboratoire de Mathematiques, Universite Paris 11, 91405 Orsay, France

Department of Pure Mathematics and Mathematical Statistics, University of CamBridge, Cambridge CB3 0WB, United Kingdom

E-mail address: P.K.Friz@statslab.cam.ac.uk

Mathematisches Institut, Universität Bonn, Endenicher Allee 60, D-53115 Bonn, Germany 